\title{
RACK1 promotes hepatocellular carcinoma cell survival via CBR1 by suppressing TNF- $\alpha$-induced ROS generation
}

\author{
SILEI ZHOU ${ }^{1,2^{*}}$, HUANLING CAO ${ }^{1,2^{*}}$, YAWEI ZHAO ${ }^{1,2}$, XINYING LI $^{1}$, JIYAN ZHANG $^{1}$, \\ CHUNMEI HOU $^{1}$, YUANFANG MA ${ }^{2}$ and QINGYANG WANG ${ }^{1}$ \\ ${ }^{1}$ Department of Molecular Immunology, Institute of Basic Medical Sciences, Beijing 100850; ${ }^{2}$ Laboratory of \\ Cellular and Molecular Immunology, Henan University, Kaifeng, Henan 475004, P.R. China
}

Received April 24, 2015; Accepted September 9, 2016

DOI: $10.3892 / \mathrm{ol} .2016 .5339$

\begin{abstract}
It has been reported that intracellular accumulation of reactive oxygen species (ROS) has a significant role in tumor necrosis factor (TNF)- $\alpha$-induced cell apoptosis and necrosis; however, the key molecules regulating ROS generation remain to be elucidated. The present study reports that knockdown of endogenous receptor for activated $\mathrm{C}$ kinase 1 (RACK1) increases the intracellular ROS level following TNF- $\alpha$ or $\mathrm{H}_{2} \mathrm{O}_{2}$ stimulation in human hepatocellular carcinoma (HCC) cells, leading to promotion of cell death. Carbonyl reductase 1 (CBR1), a ubiquitous nicotinamide adenine dinucleotide phosphate-dependent enzyme, is reported to protect cells from ROS-induced cell damage. The present study reports that RACK1 is a regulator of CBR1 that interacts with and sustains the protein stability of CBR1. Overexpression of CBR1 reverses the enhanced cell death due to RACK1 knockdown. Taken together, the results of the present study suggest that RACK1 protects HCC cells from TNF- $\alpha$-induced cell death by suppressing ROS generation through interacting with and regulating CBR1.
\end{abstract}

\section{Introduction}

Escape from tumor necrosis factor (TNF)- $\alpha$-induced cell apoptosis and necrosis is of importance in tumor development (1-3).

Correspondence to: Dr Qingyang Wang, Department of Molecular Immunology, Institute of Basic Medical Sciences, East 0749, 27 Taiping Road, Haidian, Beijing 100850, P.R. China

E-mail: tansun0532@163.com

Mr. Yuanfang Ma, Laboratory of Cellular and Molecular Immunology, Henan University, 85 Minglun Street, Kaifeng, Henan 475004, P.R. China

E-mail: mayf@henu.edu.cn

${ }^{*}$ Contributed equally

Key words: receptor for activated C kinase 1, carbonyl reductase 1, reactive oxygen species, cell death, tumor necrosis factor- $\alpha$, hepatocellular carcinoma
This process is regulated by a number of intracellular signaling pathways, including c-jun $\mathrm{N}$-terminal kinase (JNK) and $\mathrm{I} \kappa \mathrm{B}$ kinase (IKK), as well as reactive oxygen species (ROS) $(4,5)$. Extensive studies have indicated that reduced levels of oxidant stress and ROS promote malignant transformation and oncogenic growth in hepatocellular carcinoma (HCC) cells (6-9). However, the key molecules regulating ROS in HCC remain to be elucidated. It has been reported that scaffolding protein receptor for activated $\mathrm{C}$ kinase 1 (RACK1) enhances JNK activation in $\mathrm{HCC}$, leading to promotion of the malignant growth of HCC (10). Therefore, it may be assumed that RACK1 affects other aspects of HCC. RACK1 was originally identified to bind and activate protein kinase $\mathrm{C}$ and is now recognized as a multi-functional scaffold protein $(11,12)$. Evidence has indicated that RACK1 protects from oxidative stress-induced cell death in various types of cells, including fission yeasts (13), shrimp cells (14), neurons (15), HeLa cells (16) and HL60 cells (17). However, such a role for RACK1 has not been reported in HCC cells to the best of our knowledge. In the present study, it was demonstrated that RACK1 knockdown leads to increased cell death in TNF- $\alpha$-treated $\mathrm{HCC}$ cells in the presence of cycloheximide $(\mathrm{CHX})$, a protein synthesis inhibitor. Subsequently, it was observed that RACK1 knockdown promotes intracellular ROS accumulation upon TNF- $\alpha$ or $\mathrm{H}_{2} \mathrm{O}_{2}$ stimulation. A combination of co-immunoprecipitation (co-IP) and mass spectrometry analysis indicated that carbonyl reductase 1 (CBR1), a ubiquitous nicotinamide adenine dinucleotide phosphate-dependent enzyme, acts as a RACK1-interacting partner in HCC cells. CBR1 has been reported to provide protection from ROS-induced cellular damage in HCC and leukemia $(4,18)$, which suggests that CBR1 serves a role in cellular anti-oxidation. In the present study, it was reported that overexpression of exogenous CBR1 in HCC cells reverses enhanced cell death upon silencing of endogenous RACK1, which indicated that RACK1 may have a pivotal role in sustaining the protein stability of CBR1.

\section{Materials and methods}

Cell culture and treatment. Human hepatic carcinoma cell lines HepG2 and SMMC7721, and mouse embryonic liver cell line BNL CL. 2 were purchased from American Type Culture Collection (Manassas, VA, USA). Cells were cultured in Dulbecco's 
modified Eagle's medium (catalog no. 12800-058; Thermo Fisher Scientific, Inc., Waltham, MA, USA) supplemented with $10 \%$ fetal calf serum (Standard Grade; Lanzhou Bailing Biotechnology Co., Ltd., Lanzhou, China.) at $37^{\circ} \mathrm{C}$ and $5 \% \mathrm{CO}_{2}$.

Cell transfection. Small interfering RNA (siRNA) targeting human RACK1 (5'-GGATGAGACCAACTATGGAAT-3') and human CBR1 (5'-ATACGTTCACCACTCTCCCTT-3') and small hairpin RNA targeting mouse RACK1 (5'-GTCCCG AGACAAGACCATAAA-3') were designed and chemically synthesized by Shanghai GenePharma Co., Ltd. (Shanghai, China). The siRNAs were delivered into the SMMC7721 cells at $50 \%$ confluence using Lipofectamine ${ }^{\circledR}$ RNAiMAX transfection reagent (Thermo Fisher Scientific, Inc.) according to the manufacturer's protocol. Cells were transfected for $48 \mathrm{~h}$ and then analyzed for various parameters. SMMC7721 stable clones, which were a gifts from Dr Wendie Wang (Institute of Medicinal Biotechnology, Chinese Academy of Medical Sciences, Beijing, China), were selected in $600 \mu \mathrm{g} / \mathrm{ml} \mathrm{G} 418$ sulfate (catalog. no. 11811-023; Thermo Fisher Scientific, Inc.) for approximately 2 months.

Sample preparation, co-IP and western blot analysis. Cells were harvested and lysed in lysis buffer (20 mM Tris, $\mathrm{pH}$ 7.6, $250 \mathrm{mM} \mathrm{NaCl}, 1 \%$ Nonidet P-40, $3 \mathrm{mM}$ EDTA, $1.5 \mathrm{mM}$ ethylene glycol-bis( $\beta$-aminoethyl ether)-N,N,N',N'-tetraacetic acid, $10 \mu \mathrm{g} / \mathrm{ml}$ aprotinin, $10 \mathrm{mM} p$-nitrophenylphosphate, $1 \mathrm{mM} \mathrm{Na} \mathrm{VO}_{4}, 1 \mathrm{mM}$ dithiothreitol). Following clarification by centrifugation at $5,000 \times \mathrm{g}$ for $15 \mathrm{~min}$ at $4{ }^{\circ} \mathrm{C}$, cell lysates were incubated with the indicated antibodies in the presence of $30 \mu 1[50 \%(\mathrm{v} / \mathrm{v})]$ of protein A-Sepharose beads (Sigma-Aldrich; EMD Millipore, Billerica, MA, USA) at $4^{\circ} \mathrm{C}$ for $4 \mathrm{~h}$. Precipitates were washed with washing buffer [20 mM Tris (pH 7.6), $250 \mathrm{mM} \mathrm{NaCl}, 1 \%$ Nonidet P-40, 3 mM EDTA, $1.5 \mathrm{mM}$ ethylene glycol-bis( $\beta$-aminoethyl ether)-N,N,N',N'tetraacetic acid and $1 \mathrm{mM}$ phenylmethane sulfonyl fluoride) at least three times. For western blot analysis, cell lysates or co-IP samples underwent $12 \%$ SDS-PAGE for $2 \mathrm{~h}$, followed by transferal to polyvinylidene difluoride membranes for $3 \mathrm{~h}$ and blocking with 5\% nonfat milk in TBS containing $0.1 \%$ Tween-20 (TBST) for $1 \mathrm{~h}$ at room temperature. Membranes were incubated with primary antibodies against RACK1 (catalog no. 610177; BD Biosciences, San Jose, CA, USA), CBR1 (catalog no. ab4148; Abcam, Cambridge, MA, USA), $\beta$-actin (catalog no. 47778; Santa Cruz Biotechnology, Inc., Dallas, TX, USA) and GAPDH (catalog no. sc-81545; Cell Signaling Technology, Inc., Danvers, MA, USA) at a dilution of $1: 1,000$ overnight at $4^{\circ} \mathrm{C}$. Following three times washing with TBST (10 min each wash), the membranes were incubated with horseradish peroxidase-conjugated secondary antibodies at a dilution of 1:5,000 for $1 \mathrm{~h}$ at room temperature (polyclonal goat anti-rabbit or goat anti-mouse secondary antibodies; catalog no. ZB2301 and ZB2305, respectively; OriGene Technologies, Inc., Beijing, China), followed by additional washing. The membranes were subjected to exposure in the dark and the immunoreactive bands were visualized with an enhanced chemiluminescence kit (GE Healthcare Life Sciences, Chalfont, UK). Quantification of western blot were performed by using Gel-Pro Analyzer 4.0 (Media Cybernetics, Inc., Rockville, MD, USA).
Cell death assay by flow cytometry. Cells treated with $10 \mathrm{ng} / \mathrm{ml}$ TNF- $\alpha$ (R\&D Systems, Inc., Minneapolis, MN, USA) with or without $10 \mu \mathrm{g} / \mathrm{ml}$ CHX (Sigma-Aldrich; EMD Millipore), or $1 \mathrm{mM} \mathrm{H}_{2} \mathrm{O}_{2}$ for $24 \mathrm{~h}$ were digested by $0.25 \%$ trypsin for approximately $2 \mathrm{~min}$ with gentle shaking, and subsequently harvested. Following washing twice with PBS, the cell pellet was resuspended in $200 \mathrm{ml}$ PBS containing Annexin- $\mathrm{V}$ and propidium iodide (PI)/7-aminoactinomycin D (BD Biosciences) and incubated at $4^{\circ} \mathrm{C}$ for $30 \mathrm{~min}$, followed by flow cytometry assay.

ROS assay. Cells were resuspended and incubated in pre-warmed Hank's balanced salt solution (HBSS) containing $10 \mathrm{mM}$ carboxy-.2',7'-dichlorodihydrofluorescein diacetate (Thermo Fisher Scientific, Inc.) for $30 \mathrm{~min}$ at $37^{\circ} \mathrm{C}$, followed by incubation with $10 \mathrm{ng} / \mathrm{ml}$ TNF- $\alpha$ or $400 \mu \mathrm{mol} / 1 \mathrm{H}_{2} \mathrm{O}_{2}$ for $30 \mathrm{~min}$ at $37^{\circ} \mathrm{C}$. Cells were washed with HBSS twice and subjected to flow cytometry.

Statistical analysis. Cell death assay experiments were performed independently at least three times. Statistical differences between groups were assessed by Students t-test. Descriptive statistics were computed by using Excel 2007 (Microsoft Corporation, Redmond, WA, USA). P $<0.05$ was considered to indicate a statistically significant difference.

\section{Results}

Knockdown of RACK1 leads to increased cell death and ROS generation following TNF- $\alpha$ stimulation. The present study initially investigated the correlation between RACK1 and TNF- $\alpha$-induced cell death in SMMC7721 cells. SMMC7721 cells were transiently transfected with RACK1 siRNA or NC siRNA by using the iMAX delivery system. A total of $48 \mathrm{~h}$ later, the cells were treated with $10 \mathrm{ng} / \mathrm{ml} \mathrm{TNF-} \alpha$ with or without $10 \mu \mathrm{g} / \mathrm{ml} \mathrm{CHX}$ for $24 \mathrm{~h}$, followed by cell death assay with Annexin-V and PI double staining. Transfection with RACK1 siRNA dramatically decreased RACK1 protein levels (Fig. 1A), leading to an increased cell death rate compared with NC siRNA following co-treatment with TNF- $\alpha$ and CHX $\left(\mathrm{P}=2.67 \times 10^{-7}\right.$; Fig. 1B). Notably, treatment with TNF- $\alpha$ alone only caused slight cell death, and cell death was markedly induced in the presence of CHX, a pan-protein synthesis inhibitor (Fig. 1B), indicating that CHX sensitized SMMC7721 cells to TNF- $\alpha$-induced cell death. It was subsequently investigated whether RACK1 affected ROS responses to stimuli, including TNF- $\alpha$ or hydrogen peroxide. As demonstrated in Fig. 1C, knockdown of RACK1 resulted in increased ROS generation in SMMC7721 cells upon TNF- $\alpha$ and hydrogen peroxide treatment. Taken together, the results of the present study suggested that RACK1 acted as a ROS suppressor to antagonize TNF- $\alpha$-induced cell death in SMMC7721 cells.

RACK1 suppresses TNF- $\alpha$-induced cell death via CBR1. Although it was observed that knockdown of endogenous RACK1 promoted ROS generation upon TNF- $\alpha$ and hydrogen peroxide stimulation, the molecular mechanism(s) by which RACK1 affected ROS remain to be elucidated. Subsequently, a co-IP was performed, followed by mass spectrometry analysis (performed by National Center of Biomedical Analysis, Beijing, 
A

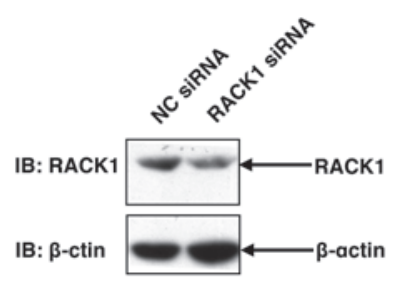

B

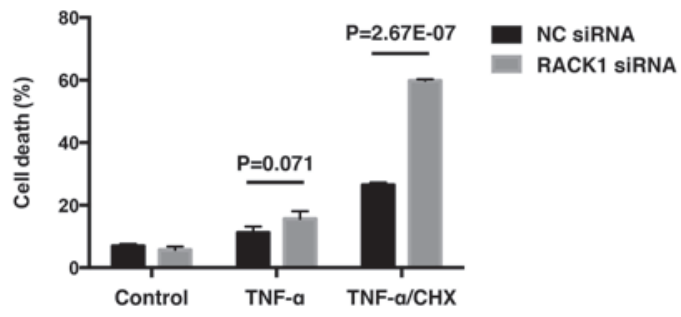

C

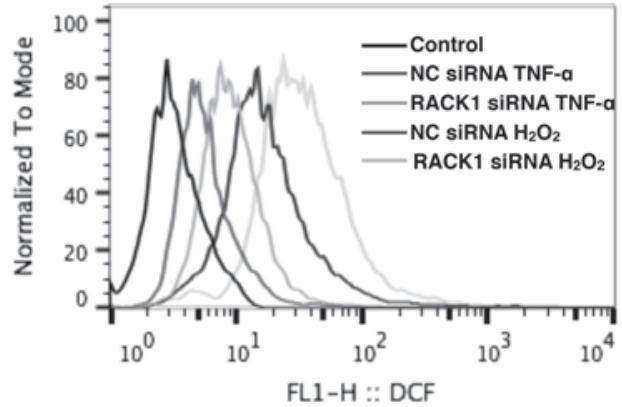

Figure 1. Knockdown of RACK1 promotes cell death and ROS generation. (A) SMMC7721 cells were transiently transfected with NC siRNA or RACK1 siRNA. Cell lysates were subjected to western blot analysis. (B) SMMC7721 cells were transiently transfected with NC siRNA or RACK1 siRNA. A total of $48 \mathrm{~h}$ later, cells were stimulated with $10 \mathrm{ng} / \mathrm{ml} \mathrm{TNF}-\alpha$ with or without $10 \mu \mathrm{g} / \mathrm{ml} \mathrm{CHX}$, followed by cell death staining and flow cytometry assays. Cell death was determined by fluorescein-Annexin- $\mathrm{V}$ and propidium iodide double staining, and the results of three independent experiments are presented as the mean \pm standard deviation; $n=3$. (C) SMMC7721 cells were transiently transfected with NC siRNA or RACK1 siRNA. Prior to flow cytometry assays, cells were treated with TNF- $\alpha$ or hydrogen peroxide and incubated with $10 \mathrm{mM} \mathrm{5(6)-carboxy-.2',7'-dichlorodihydrofluorescein} \mathrm{diacetate} \mathrm{green} \mathrm{probes.} \mathrm{Intracellular}$ ROS levels were measured by flow cytometry. RACK1, receptor for activated C kinase 1; ROS, reactive oxygen species; NC, negative control; siRNA, small interfering RNA; TNF, tumor necrosis factor; CHX, cycloheximide; IB, immunoblotting.

A

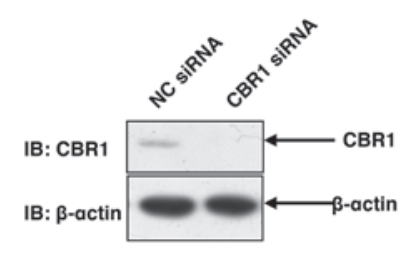

C

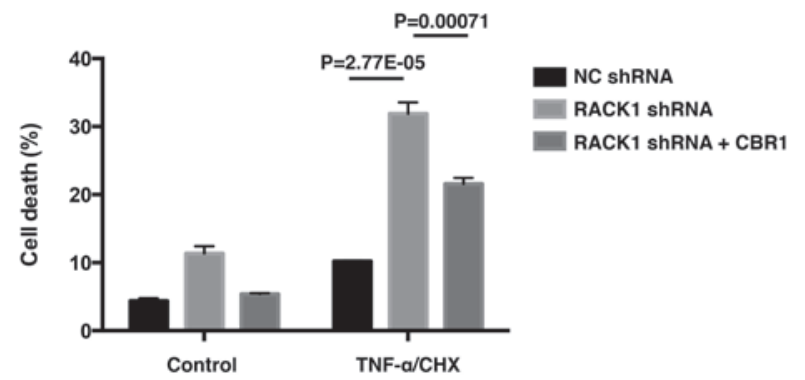

B

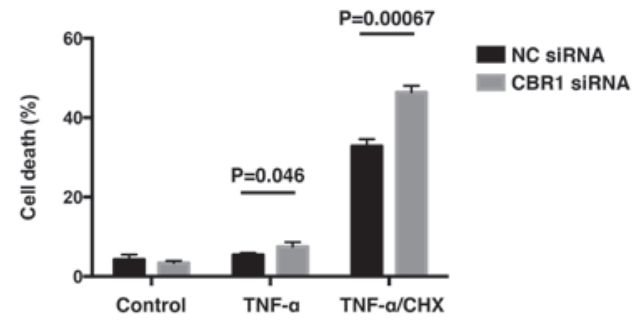

D

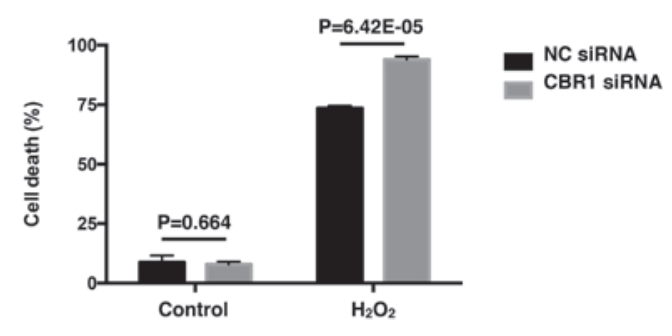

Figure 2. CBR1 promotes cell survival and reverses the enhanced cell death caused by RACK1 knockdown. (A) SMMC7721 cells were transiently transfected with NC siRNA or CBR1 siRNA. The amounts of CBR1 protein were examined by western blot analysis with the indicated antibodies. (B) SMMC7721 cells were transiently transfected with NC siRNA or CBR1 siRNA. Cells were stimulated with $10 \mathrm{ng} / \mathrm{ml}$ TNF- $\alpha$ with or without $10 \mu \mathrm{g} / \mathrm{ml} \mathrm{CHX,} \mathrm{followed} \mathrm{by} \mathrm{cell}$ death staining and flow cytometry assays. Cell death was determined by FITC-Annexin-V and PI double staining. (C) Control clone cells and RACK1 stably silenced clone cells were transfected with GFP or GFP-CBR1 vectors. Cells were stimulated with $10 \mathrm{ng} / \mathrm{ml} \mathrm{TNF}-\alpha$ and $10 \mu \mathrm{g} / \mathrm{ml} \mathrm{CHX} \mathrm{for} 24 \mathrm{~h}$, followed by flow cytometry assays. GFP positive cells were gated and cell death was analyzed with phycoerythrin-Annexin-V and 7-aminoactinomycin D double staining. (D) SMMC7721 cells transfected with NC siRNA or CBR1 siRNA were stimulated with $1 \mathrm{mM} \mathrm{H}_{2} \mathrm{O}_{2}$ for $24 \mathrm{~h}$, followed by cell death staining and flow cytometry assays. Cell death was determined by FITC-Annexin-V and PI double staining. Results are representative of three independent experiments and are presented as the mean \pm standard deviation; $n=3$. CBR1, carbonyl reductase 1; RACK1, receptor for activated C kinase 1; NC, negative control; siRNA, small interfering RNA; shRNA, short hairpin RNA; TNF, tumor necrosis factor; CHX, cycloheximide; IB, immunoblotting; FITC, fluorescein isothiocyanate; PI, propidium iodide; GFP, green fluorescent protein. 
A

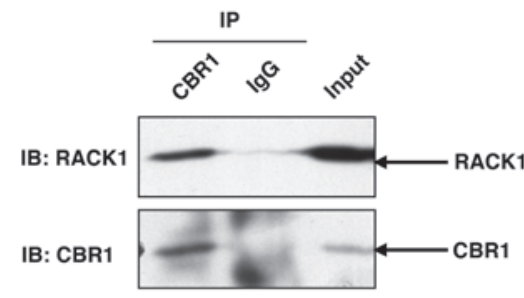

B

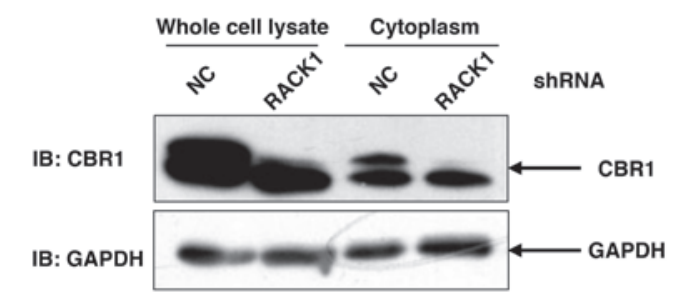

C

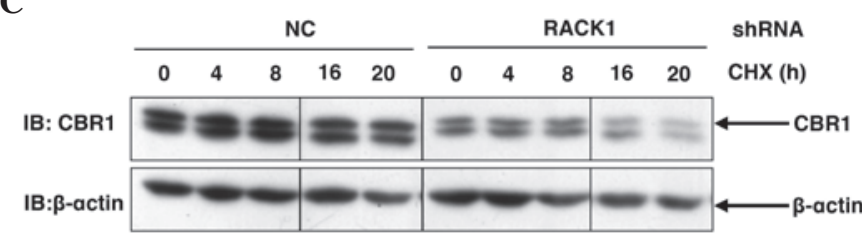

D

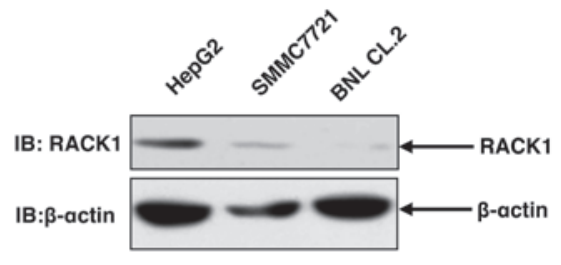

$\mathbf{E}$

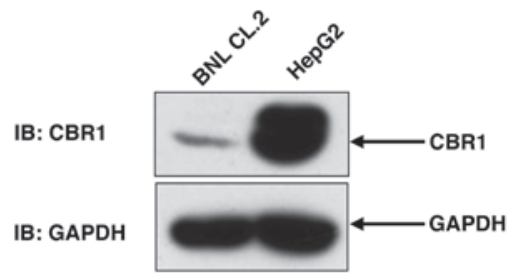

$\mathbf{F}$

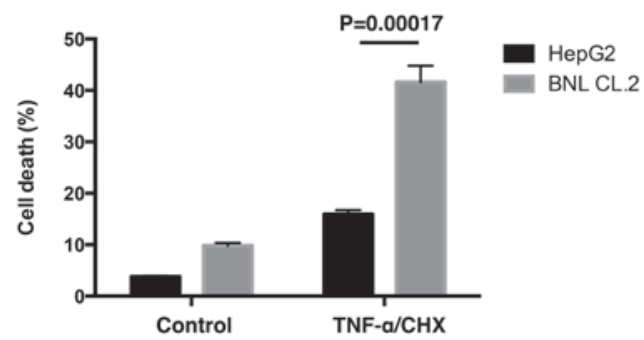

Figure 3. RACK1 binds to CBR1 and sustains the protein stability of CBR1. (A) SMMC7721 cells were lysed and the lysates were subjected to immunoprecipitation with an anti-CBR1 antibody or a control antibody. Following immunoprecipitation, western blot analysis was performed. (B) Control clone cells and RACK1 stably silenced clone cells were lysed and subjected to western blot analysis. (C) Control clone cells and RACK1 stably silenced clone cells were treated with CHX for various durations, followed by western blot analysis. (D and E) HepG2 cells, SMMC7721 cells and BNL CL.2 cells were lysed and subjected to western blot analysis. (F) Following TNF- $\alpha / \mathrm{CHX}$ stimulation, HepG2 cells and BNL CL. 2 cells were subjected to flow cytometry assays. Cell death was determined by fluorescein isothiocyanate-Annexin-V and propidium iodide double staining, and the results of three independent experiments are presented as the mean \pm standard deviation; $n=3$. RACK1, receptor for activated $\mathrm{C}$ kinase 1; CBR1, carbonyl reductase 1; CHX, cycloheximide; TNF, tumor necrosis factor; IP, immunoprecipitation; NC, negative control; IB, immunoblotting; shRNA, short hairpin RNA.

China) to identify RACK1-interacting partners (data not shown). Among the candidates of RACK1-interacting proteins obtained, CBR1 was notable due to its close association with ROS. CBR1 was considered to be a ROS suppressor, as it had been reported to protect cells from cytotoxic drug-triggered cell death in doxorubicin-treated $\mathrm{HCC}$ cells and $\mathrm{As}_{2} \mathrm{O}_{3}$-treated leukemia cells $(4,18)$. Therefore, the functions of CBR1 were investigated in TNF- $\alpha$-treated SMMC7721 cells. As expected, transfection with CBR1 siRNA markedly decreased the protein level of CBR1 (Fig. 2A), leading to increased percentages of cell death induced by TNF- $\alpha(\mathrm{P}=0.046)$ and TNF- $\alpha /$ CHX (P=0.00067; Fig. 2B). Subsequently, the present study employed RACK1 stably silenced single clones screened from SMMC7721 cells to investigate whether CBR1 affected the enhanced cell death caused by RACK1 knockdown. As expected, overexpression of green fluorescent protein (GFP)-CBR1 reversed the enhanced cell death in the RACK1 stably silenced clone, at least partially, compared with the clone overexpressing GFP ( $\mathrm{P}=0.00071$; Fig. 2C). Cell death upon hydrogen peroxide stimulation was also investigated in HCC cells. Rather than apoptotic cell death, hydrogen peroxide stimulation caused a more necrotic cell death than TNF- $\alpha /$ CHX stimulation (data not shown). However, hydrogen peroxide caused $\sim 80 \%$ cell death, and knockdown of CBR1 elevated cell death up to $>90 \%\left(\mathrm{P}=6.42 \times 10^{-5}\right.$; Fig. 2D). Thus, the results of the present study suggested RACK1 may exert its protecting function via CBR1.

RACK1 promotes the protein stability of CBR1. To address whether RACK1 affected the functioning of CBR1, it was necessary to address whether and how RACK1 regulates CBR1. Initially, the present study briefly addressed the interaction between RACK1 and CBR1 in vivo. As shown in Fig. 3A, when endogenous CBR1 was immunoprecipitated from SMMC7721 cells with CBR1 antibody, RACK1 was detected in the precipitant, suggesting that RACK1 was able to interact with CBR1 in SMMC7721 cells (Fig. 3A). Furthermore, in SMMC7721 RACK1 stably silenced single clones, CBR1 protein levels exhibited a marked decrease compared to control clones (Fig. 3B), suggesting that RACK1 potentially regulated the protein stability of CBR1. For further investigation, the present study examined the degradation of CBR1 in HCC cells in RACK1 stably silenced clones and control clones upon CHX treatment for various time courses. CBR1 protein exhibited a decrease at 16 and $20 \mathrm{~h}$ subsequent to $\mathrm{CHX}$ treatment in HCC clones with RACK stable knockdown but 
exhibited no change in control HCC clones (Fig. 3C). It has previously been reported that RACK1 exhibited higher expression in HCC cells compared to 'normal' hepatocytes (10). As expected, an increased level of RACK1 protein was detected in SMMC7721 cells and HepG2 cells, as compared to BNL CL.2 mouse embryonic liver cells (Fig. 3D). Consistent with the idea that RACK1 promotes the stability of CBR1 protein, HepG2 cells exhibited an increased level of CBR1 protein compared with BNL CL.2 cells (Fig. 3E). This finding is consistent with a previous report in which CBR1 demonstrated overexpression in $56(72 \%)$ out of 78 human HCC tissues (6). Notably, upon TNF- $\alpha /$ CHX stimulation, BNL CL. 2 cells exhibited a marked increase in cell death compared to HepG2 cells $(\mathrm{P}=0.00017$; Fig. 3F). These results indicate that upregulation of RACK1 together with CBR1 may have a significant role in the transformation of normal liver cells to malignant cells.

\section{Discussion}

In the present study, it was initially confirmed that RACK1 protected HCC cells from TNF- $\alpha$-induced cell death. However, it was observed that TNF- $\alpha$ alone only caused slightly enhanced cell death. It has been reported that metabolic inhibitors, including actinomycin D or CHX, dramatically sensitized HCC cells to TNF- $\alpha$-induced cell death due to their ability to inhibit the de novo synthesis of anti-apoptotic proteins (19). Following co-treatment with TNF- $\alpha$ and CHX, knockdown of RACK1 led to markedly enhanced cell death in SMMC7721 cells. This may be attributed to increased ROS generation upon TNF- $\alpha$ stimulation. Hydrogen peroxide stimulation also increased ROS generation and cell death. Treatment of HCC cells with TNF- $\alpha / \mathrm{CHX}$ is frequently used to mimic acute fulminant hepatitis (20). Therefore, further investigation of the mechanisms by which RACK1 affects the cell death of HCC may support novel clinic treatments for acute fulminant hepatitis.

Among the signaling pathways mediating TNF- $\alpha$ signal transduction, three are considered to regulate the process of TNF- $\alpha$-induced cell death: Mitogen-activated protein kinase, nuclear factor (NF)- $\mathrm{BB}$ and ROS. In a previous study, JNK activity, which is frequently involved in promotion of cell death, was downregulated by the knockdown of RACK1 (10). The NF- $\mathrm{KB}$ signaling pathway is considered to be crucial to anti-TNF- $\alpha$-induced cell death, as TNF- $\alpha$-induced NF- $\kappa B$ activation induces the expression of numerous anti-apoptotic genes $(21,22)$. However, it has been reported IKK $\beta$ depletion in hepatocytes only causes slightly increased sensitivity of cells to lipopolysaccharide challenge (23), which indicates that the effects of the NF- $\mathrm{kB}$ signaling pathway are compensated by other factors in this process. In the present study, CBR1 was identified as regulating TNF- $\alpha$-induced HCC cell death. Knockdown of CBR1 gave rise to enhanced cell death following TNF- $\alpha / \mathrm{CHX}$ stimulation, and overexpression of CBR1 in RACK1 stably silenced single clone reversed enhanced cell death due to the downregulation of RACK1. These data suggested RACK1 exerted its protecting function via CBR1. Further investigation revealed that RACK1 and CBR1 were involved in the same complex, indicating that RACK1 is bound to CBR1. It should be noted that the amount of CBR1 protein in RACK1 stably silenced clone markedly decreased compared to the control, suggesting that RACK1 sustained the protein level of CBR1. Further experiments confirmed this observation. As RACK1 and CBR1 have been confirmed to be highly expressed in human HCC by previous studies $(6,10)$, the results of the present study suggested that the levels of RACK1 may be positively correlated with those of CBR1 in human HCC. Additionally, compared to non-malignant BNL CL.2 cells, malignant HepG2 cells exhibited increased protein levels of RACK1 and CBR1, and increased resistance to TNF- $\alpha$-induced cell death. Therefore, the positive association between RACK1 and CBR1 may act in the malignant transformation of normal liver cells to malignant cells. However, these theories require further investigation.

In conclusion, the results of the present study have confirmed that the increased expression of RACK 1 in HCC cell lines causes increased protein levels of CBR1 and a reduced ROS response, thereby leading to increased resistance to TNF- $\alpha$-induced cell death.

\section{Acknowledgements}

The present study was supported by grants from the National Natural Science Foundation of China (grant no., 81472736 to JZ) and Innovation Foundation (grant no., 2015cxjj-011).

\section{References}

1. Wajant H: The role of TNF in cancer. Results Probl Cell Differ 49: 1-15, 2009.

2. Ivanov VN, Bhoumik A and Ronai Z: Death receptors and melanoma resistance to apoptosis. Oncogene 22: 3152-3161, 2003.

3. Wang S and El-Deiry WS: TRAIL and apoptosis induction by TNF-family death receptors. Oncogene 22: 8628-8633, 2003.

4. Seki E, Brenner DA and Karin M: A liver full of JNK: Signaling in regulation of cell function and disease pathogenesis, and clinical approaches. Gastroenterology 143: 307-320, 2012.

5. Schwabe RF and Brenner DA: Mechanisms of liver injury. I. TNF-alpha-induced liver injury: Role of IKK, JNK, and ROS pathways. Am J Physiol Gastrointest Liver Physiol 290: G583-G589, 2006.

6. Tak E, Lee S, Lee J, Rashid MA, Kim YW, Park JH, Park WS Shokat KM, Ha J and Kim SS: Human carbonyl reductase 1 upregulated by hypoxia renders resistance to apoptosis in hepatocellular carcinoma cells. J Hepatol 54: 328-339, 2011.

7. Kim YS, Seo HW and Jung G: Reactive oxygen species promote heat shock protein 90-mediated HBV capsid assembly. Biochem Biophys Res Commun 457: 328-333, 2015.

8. Lin B, Tan X, Liang J, Wu S, Liu J, Zhang Q and Zhu R: A reduction in reactive oxygen species contributes to dihydromyricetin-induced apoptosis in human hepatocellular carcinoma cells. Sci Rep 4: 7041, 2014.

9. Xia L, Mo P, Huang W, Zhang L, Wang Y, Zhu H, Tian D, Liu J, Chen Z, Zhang Y, et al: The TNF- $\alpha /$ ROS/HIF-1-induced upregulation of FoxMI expression promotes HCC proliferation and resistance to apoptosis. Carcinogenesis 33: 2250-2259, 2012.

10. Guo Y, Wang W, Wang J, Feng J, Wang Q, Jin J, Lv M, Li X, Li Y, Ma Y, et al: Receptor for activated C kinase 1 promotes hepatocellular carcinoma growth by enhancing mitogen-activated protein kinase kinase 7 activity. Hepatology 57: 140-151, 2013.

11. Ron D and Mochly-Rosen D: An autoregulatory region in protein kinase C: The pseudoanchoring site. Proc Natl Acad Sci USA 92: 492-496, 1995.

12. Gibson TJ: RACK1 research - ships passing in the night? FEBS Lett 586: 2787-2789, 2012.

13. Mos M, Esparza-Franco MA, Godfrey EL, Richardson K, Davey J and Ladds G: The role of the RACK1 ortholog Cpc2p in modulating pheromone-induced cell cycle arrest in fission yeast. PLoS One 8: e65927, 2013. 
14. Saelee N, Tonganunt-Srithaworn M, Wanna W and Phongdara A: Receptor for activated $\mathrm{C}$ kinase-1 protein from Penaeus monodon (Pm-RACK1) participates in the shrimp antioxidant response. Int J Biol Macromol 49: 32-36, 2011.

15. Ma J, Wu R, Zhang Q, Wu JB, Lou J, Zheng Z, Ding JQ and Yuan Z: DJ-1 interacts with RACK1 and protects neurons from oxidative-stress-induced apoptosis. Biochem J 462: 489-497, 2014.

16. Polosukhina D, Singaravelu K and Padanilam BJ: Activation of protein kinase $\mathrm{C}$ isozymes protects LLCPK1 cells from $\mathrm{H} 2 \mathrm{O} 2$ induced necrotic cell death. Am J Nephrol 23: 380-389, 2003.

17. Korchak HM and Kilpatrick LE: Roles for beta II-protein kinase $\mathrm{C}$ and RACK1 in positive and negative signaling for superoxide anion generation in differentiated HL60 cells. J Bio Chem 276: 8910-8917, 2001.

18. Jang M, Kim Y, Won H, Lim S, K R J, Dashdorj A, Min YH, Kim SY, Shokat KM, Ha J and Kim SS: Carbonyl reductase 1 offers a novel therapeutic target to enhance leukemia treatment by arsenic trioxide. Cancer Res 72: 4214-4224, 2012.
19. Okano H, Shiraki K, Inoue H, Kawakita T, Yamanaka T, Deguchi M, Sugimoto K, Sakai T, Ohmori S, Fujikawa K, et al: Cellular FLICE/caspase-8-inhibitory protein as a principal regulator of cell death and survival in human hepatocellular carcinoma. Lab Invest 83: 1033-1043, 2003.

20. Pathil A, Warth A, Chamulitrat W and Stremmel W: Comparison of different bile acid-phospholipid conjugates in acute hepatitis. Eur J Clin Invest 42: 130-138, 2012.

21. Van Antwerp DJ, Martin SJ, Kafri T, Green DR and Verma IM: Suppression of TNF-alpha-induced apoptosis by NF-kappaB. Science 274: 787-789, 1996.

22. Tang G, Minemoto Y, Dibling B, Purcell NH, Li Z, Karin M and Lin A: Inhibition of JNK activation through NF-kappaB target genes. Nature 414: 313-317, 2001.

23. Maeda S, Chang L, Li ZW, Luo JL, Leffert $\mathrm{H}$ and Karin M: IKKbeta is required for prevention of apoptosis mediated by cell-bound but not by circulating TNFalpha. Immunity 19: 725-737, 2003. 